\title{
Reflets
}

Revue d'intervention sociale et communautaire

\section{Le secteur « Femmes » de l'Entente Canada-communauté-Ontario : des priorités pour l'avenir} Élaine Dery

Volume 9, numéro 1, printemps 2003

Le genre en contexte : pratiques sociales et représentations

URI : https://id.erudit.org/iderudit/010871ar

DOI : https://doi.org/10.7202/010871ar

Aller au sommaire du numéro

Éditeur(s)

Reflets : Revue ontaroise d'intervention sociale et communautaire

ISSN

1203-4576 (imprimé)

1712-8498 (numérique)

Découvrir la revue

Citer cet article

Dery, É. (2003). Le secteur « Femmes » de l’Entente

Canada-communauté-Ontario : des priorités pour l'avenir. Reflets, 9(1),

231-235. https://doi.org/10.7202/010871ar
Résumé de l'article

Cet article présente brièvement les priorités du secteur « Femmes " liées à l'Entente Canada-communauté-Ontario. Il donne également un aperçu de la manière dont ces priorités ont été établies par les femmes et les groupes de femmes francophones grâce à un processus de consultation provinciale mené par la Table féministe francophone de concertation provinciale de l'Ontario.
Tous droits réservés @ Reflets : Revue ontaroise d'intervention sociale et communautaire, 2002
Ce document est protégé par la loi sur le droit d'auteur. L'utilisation des services d'Érudit (y compris la reproduction) est assujettie à sa politique d'utilisation que vous pouvez consulter en ligne.

https://apropos.erudit.org/fr/usagers/politique-dutilisation/ 


\section{Le secteur «Femmes » de l'Entente Canada-communauté-Ontario : des priorités pour l'avenir}

Cet article présente brièvement les priorités du secteur « Femmes " liées à l'Entente Canadacommunauté-Ontario. Il donne également un aperçu de la manière dont ces priorités ont été établies par les femmes et les groupes de femmes francophones grâce à un processus de consultation provinciale mené par la Table féministe francophone de concertation provinciale de l'Ontario.

\section{Élaine Dery}

Depuis 1995, la Table féministe francophone de concertation provinciale de l'Ontario représente le mouvement des femmes francophones ainsi que le secteur "Femmes " au sein du Comité de direction de l'Entente Canada-communauté-Ontario (Entente). Grâce à l'Entente, le gouvernement fédéral soutient la communauté minoritaire de langue française en Ontario pour lui permettre de prendre en charge son développement et son épanouissement. Une centaine d'organismes francophones œuvrant à l'essor de la communauté de langue française reçoivent du financement de l'Entente, notamment les groupes de femmes francophones composant le secteur "Femmes ". Bien que l'Entente actuelle arrivera à échéance en 2004, le secteur "Femmes » a récemment identifié ses priorités d'intervention sur la scène ontarienne. 


\section{Une vision pour l'avenir}

Des efforts de concertation et de consultation déployés dans les diverses régions de l'Ontario ont permis à la communauté d'expression française de se doter du plan stratégique communautaire : L'Ontario français : uni, solidaire et engagé. ${ }^{1}$ Depuis avril 2002, l'Ontario français bénéficie, en effet, de cet outil privilégié de développement et d'épanouissement. Ce plan précise la vision, les valeurs et les axes de développement guidant dorénavant les interventions des groupes communautaires francophones de l'Ontario. Les seize secteurs composant l'Entente ${ }^{2}$ ont été invités à s'inspirer du plan stratégique communautaire pour élaborer des plans opérationnels, plans visant à établir les résultats que chacun des secteurs entend atteindre d'ici 2004.

\section{Plan opérationnel du secteur « Femmes »}

C'est dans ce cadre que le plan opérationnel du secteur « Femmes » a été élaboré puis adopté par le mouvement des femmes francophones. Ce plan sectoriel est le résultat d'une démarche de concertation et de consultation menée auprès des groupes de femmes et de femmes francophones de la province au printemps et à l'été 2002. À cette occasion, les groupes et les femmes ont été invités à prendre connaissance du plan stratégique communautaire afin de vérifier si l'ensemble des besoins et des préoccupations du secteur "Femmes " y étaient bien représentés. Ils ont aussi été conviés à participer à l'élaboration du plan conformément aux principes de la gestion axée sur les résultats; principes commandant d'identifier clairement :

1. ce que les actions entreprises par le secteur "Femmes" produiront comme changements dans la communauté francophone; 
2. les produits ou services qui émergeront de ces résultats;

3. la manière par laquelle les progrès seront évalués;

4. les liens avec les axes de développement et les objectifs du plan stratégique de la communauté.

Ce plan sectoriel vise, à très long terme, les résultats suivants : une consolidation du secteur "Femmes " en Ontario français; une meilleure efficacité des groupes de femmes francophones à défendre leurs droits, leurs intérêts et leurs préoccupations sur les scènes économique, politique et sociale de la province ontarienne; une représentation équitable des femmes dans les diverses instances décisionnelles tant locales, régionales que provinciales; une meilleure intégration des droits et des préoccupations des femmes francophones dans les politiques gouvernementales; l'atteinte de l'égalité entre les sexes dans la société canadienne.

Pour parvenir à ces résultats, cinq secteurs d'intervention ont été suggérés pour lesquels quatre leaders ont été identifiés : santé - Comité FEMMES pour la santé; politique — Table féministe francophone de concertation provinciale de l'Ontario; économique - Table féministe francophone de concertation provinciale de l'Ontario; immigration - Mouvement ontarien des femmes immigrantes francophones; violence - Action ontarienne contre la violence faite aux femmes. ${ }^{3}$

\section{Des priorités définies par les femmes}

Ce plan sectoriel reflète les priorités établies précédemment dans la planification stratégique du secteur "Femmes " que tous les groupes de femmes francophones consultés ont été invités à examiner afin de vérifier si elle répondait à leurs besoins et à leurs préoccupations.

Les priorités du secteur "Femmes » ont été déterminées à la suite d'un processus de consultation mené entre le 31 mars et le 7 juin 2001.Au-delà de trois cent quatre-vingt femmes issues de groupes de femmes, du milieu associatif francophone ou reconnues 
en tant que leaders de diverses collectivités ont pris part aux douze rencontres régionales et provinciales de consultation. Chacune des rencontres a permis aux participantes de se familiariser avec l'Entente Canada-communauté-Ontario et le processus de planification stratégique de la communauté d'expression française. Le comité de coordination de la Table féministe francophone de concertation provinciale de l'Ontario a contribué à l'analyse des données recueillies. Plus de quarante-huit groupes de femmes ou communautaires ont validé le rapport de consultation du secteur «Femmes » en juillet 2001.

\section{Planification stratégique du secteur « Femmes »}

La planification stratégique du secteur «Femmes » repose sur le constat que, même si l'égalité devant la loi est reconnue par la Charte canadienne des droits et libertés, des inégalités entre les femmes et les hommes subsistent toujours sur les plans économique, juridique et social. Socialement désavantagées, les femmes francophones de l'Ontario doivent lutter pour la reconnaissance de leurs droits à l'égalité ( lutte qui inclut la reconnaissance de leurs droits linguistiques). Partant de ce constat, cette planification stratégique établit clairement l'importance d'améliorer les conditions de vie des femmes francophones en favorisant l'atteinte des droits à l'égalité. Dans ce contexte, les groupes communautaires du secteur «Femmes » de l'Ontario français ont privilégié l'élimination de la pauvreté et de la violence exercée envers les femmes.

Les actions menées par les groupes de femmes francophones de l'Ontario visent à éliminer la pauvreté chez les femmes en favorisant l'émergence de mesures permettant la création d'un filet de sécurité sociale et l'obtention d'un revenu décent ( salaire minimum, revenu de citoyenneté, pensions de vieillesse et de survivante décentes, programme d'assurance-emploi plus équitable pour les femmes et les travailleuses précaires, reconnaissance du travail des femmes au foyer et des aidantes, insertion du travail des 
femmes dans le PIB du pays, etc.). Le secteur «Femmes » reconnait également l'importance pour les femmes d'avoir :l'accès à l'emploi, des conditions de travail adéquates ( normes minimales d'emploi décentes, avantages sociaux, accès à la syndicalisation, emplois stables, loi sur l'équité salariale, milieu de travail flexible pour répondre aux exigences de la conciliation des activités familiales et professionnelles, etc.), des services de garde accessibles, la reconnaissance des acquis et des diplômes étrangers et l'accès à une éducation en français.

Le secteur "Femmes " juge la violence exercée envers les femmes - violence conjugale et agressions sexuelles — comme un obstacle majeur à l'atteinte des droits à l'égalité pour les femmes francophones. Pour favoriser son élimination, il prône la mise en œuvre de mesures, telles le développement de services en français ( permanence des fonds des initiatives instaurés en 1997, restructuration des services en agression sexuelle dans l'Est ontarien, ajout de maisons d'hébergement francophones, redressement financier des services existants, allocation de ressources pour assurer l'autonomie des services offerts en français, augmentation ou implantation de ressources dans le Nord, le Nord-Ouest, dans le Sud-Ouest et pour les femmes vivant avec un handicap ) et le développement de matériel en langue française (outils en français adaptés aux réalités des femmes francophones ontariennes pour mener des campagnes de sensibilisation, former des intervenantes, intervenir dans les écoles, etc.).

\section{Notes}

1. Ce document est disponible sur le site Internet : www.entente.ca

2. Les secteurs de l'Entente représentent des champs d'activités ou des groupes identitaires spécifiques œuvrant sur des volets de la vie de la communauté francophone.

3. Pour obtenir les détails des divers résultats visés, il est possible de se procurer le plan sectoriel du secteur «Femmes » sur le site Internet : www.entente.ca 